\title{
The Associations Between Preceptor Team Lead Relationships and Resident Wellness in an Academic Medicine Setting: An Exploratory Study
}

\author{
Cesar A. Gonzalez, PhD | Natalie E Gentile, MD | Kurt B Angstman, MD | Julia R Craner, PhD | Robert P. \\ Bonacci, MD
}

Published: 6/22/2017 | DOI: 10.22454/PRiMER.2017.1.5

\section{Abstract}

Background: Lack of wellness among physicians has been associated with increased risk for physical and mental illness, interpersonal discord, and occupational liability. In academic primary care practices, physician wellness and self-care behaviors have been associated with improved patient outcomes. With the increase in team-based care structures in primary care clinics and residencies there may be opportunities to promote wellness among primary clinicians, particularly among resident physicians who are at increased risk for decreased well being. The primary objective of the study was to test an a priori hypothesis that family medicine residents' perception of support from preceptor team leads would be associated with wellbeing. A secondary objective of the study was to test a post hoc hypothesis that examined whether the relationship between residents' perception of support from their preceptor team leads would be associated with residents' well being, while controlling for self-care behaviors.

Methods: Our study utilized a prospective cross-sectional design with purposive sampling to survey family medicine residents. Data were collected in February 2016. The survey was sent out to 58 family medicine residents across three family medicine residencies at Mayo Clinic. The survey response rate was 55\% ( $n=32)$; Ten (31.3\%) residents reported being in their PGY-1, 11 (34.4\%) in PGY-2; and 11 (34.4\%) in PGY-3; participants included 19 (59.4\%) women and 13 (40.6\%) men. The Brief Resident Wellness Profile (BRWP) was utilized to assess family medicine residents' perceived sense of professional accomplishment and mood in the past week.

Results: In bivariate correlational analyses, increased perception of support from preceptor team leads $(r=.40, P<.01)$ and reporting a male gender $(r=.43, P<.01)$ was associated with increased resident wellness. In exploratory multivariate analysis, results suggested that while controlling for gender, frequency of self-care behaviors, and perceived preceptor team lead support, a one-point change on rating of perceived team leader support is associated with a 1.69 increase in resident wellness score on the BRWP.

Conclusions: Our results provide preliminary evidence to support the relationship between preceptor team lead support and resident wellness in team-based care, above and beyond the impact that self-behaviors have on wellness. Our findings suggest evidence for the subsequent study of the impact of preceptor team lead relationship quality on resident wellness.

\section{Introduction}

Our review of published abstracts suggested a statistically significant upward trend in the frequency of published peer-reviewed articles related to resident physicians' wellness and burnout (Figure 1). In 2014, nearly 12 years after a 
significant increase in the frequency of peer reviewed articles on the topic of resident wellness, and after the first published expert consensus statement specific to identifying depression and suicide among physicians, ${ }^{1}$ the Quadruple Aim was introduced. ${ }^{2,3}$ The Quadruple Aim promotes the goal of supporting increased quality health care and access and reducing health care costs by prioritizing the health and wellness of health care clinicians and staff. ${ }^{3}$ Lack of wellness among physicians has been associated with increased risk for physical and mental illness, interpersonal discord, and occupational liability while self-care behaviors were directly associated with physician well being as well as professional accomplishment and satisfaction. ${ }^{4-8}$ Furthermore, burnout and dissatisfaction among physicians is linked with the future reduction of work hours spent on providing patient care and is estimated to account for approximately $1 \%$ of the reduction in the professional effort of the physician workforce in the United States. ${ }^{9,10}$ In conjugation with the projection that estimates that by 2025 there will be shortage between 14,900 and 35,600 physicians in primary care, ${ }^{11}$ identifying and resolving issues that may contribute to burnout and dissatisfaction represents an important avenue for research and improvement efforts.

In an attempt to meet the Triple Aim, there has been an increase in team-based care structures in primary care clinics and residencies. ${ }^{12}$ In nonacademic primary care clinics, team-based care is linked with increased career satisfaction and physician wellness; accordingly, self-care behaviors are associated with improved patient health outcomes; ${ }^{13-16}$ self-care behaviors are directly associated with physician wellbeing in addition to increased sense of professional accomplishment and satisfaction. ${ }^{4-8}$ Among academic medical practices, dissatisfaction with clinical faculty is associated with burnout. ${ }^{17}$ Surprisingly, the associations between wellness and self-care behaviors among physicians-in-training in team-based care in academic clinical practice has not been evaluated. However, prior research, suggests that there may be opportunities to promote wellness among health care clinicians through team-based care. ${ }^{16,18,19}$ In academic primary care practices, however, it remains unclear how to best promote wellness in a resident physician population. ${ }^{20}$ In addition, specific factors that are associated with wellness in a team-based care environment, such as clinical faculty support, have not yet been examined.

The aim of this study was to test an exploratory hypothesis about whether family medicine residents' perception of support from their team preceptor would predict wellbeing, above and beyond self-care behaviors.

\section{Methods}

Our study utilized a prospective cross-sectional design with purposive sampling to survey family medicine residents' demographics, self-care behaviors, perceived support from team preceptor, and their associations to resident wellness. Data for this study were collected in February 2016 across three family medicine residencies at Mayo Clinic in Minnesota, Wisconsin, and Florida. The study was deemed exempt by the Institutional Review Board at Mayo Clinic.

The survey was sent out to 58 family medicine residents across three family medicine residencies at Mayo Clinic. Out of the 39 residents that accessed the survey, 32 residents completed all measures. The response rate to our survey was $55 \%$. Ten (31.3\%) residents reported being in their PGY-1, 11 (34.4\%) in PGY-2; and 11 (34.4\%) in PGY-3; participants included 19 (59.4\%) women and $13(40.6 \%)$ men.

Measures

Self-Care Behaviors. The Mindful Self-Care Scale (MSCS) ${ }^{21}$ is a 33-item scale that was administered to assess level of self-care behaviors among residents. The MSCS asked residents to rate the frequency of self-care behavior in the past week. Responses to the items are on a 5-point Likert scale and range from 1 (Never or 0 days) to 5 (Regularly or 6 to 7 days); relevant reverse coding was implemented. Responses to all items were totaled, with higher scores representing increased frequency of self-care behaviors.

Team Preceptor Support. A five-item questionnaire was developed to assess levels of family residents' perceived level of team preceptor trust and support. The items were on a 7-point Likert scale from 1 (Strongly Disagree) to 7 (Strongly Agree) and included statements such as, "My team leader and I have a good working relationship." and "I trust my team leader." Responses to the five items were averaged, with higher scores indicating higher levels of perceived trust and support from the family medicine residents' team leader. 
Resident Wellness. The Brief Resident Wellness Profile (BRWP) ${ }^{22}$ was utilized to assess family medicine residents' perceived sense of professional accomplishment and mood in the past week. The BRWP is a 6-item instrument that asks residents to rate themselves on a 5-point Likert scale ranging from 1 (never) to 5 (always). Responses to all six items were summed to generate a total score, with higher scores on the BRWP indicating higher levels of resident wellness. The BRWP was chosen because the brief nature of this instrument minimizes time burden for participants and it is available for use without additional costs. Further, the BRWP assesses general wellbeing rather than a single specific construct

\section{Results}

\section{Data Analysis}

Descriptive and psychometric properties of all measures are included in Table 1. Results of the bivariate correlations between gender, postgraduate year, self-care behaviors, and perceived support from team preceptor, and resident wellness are on Table 2. Unstandardized $(b)$ and standardized $(\beta)$ regression coefficients are reported. We also reported two-tailed $p$ values (two-sided), with values less than .05 as the threshold for statistical significance. Evaluation of the statistical assumptions for multiple regression did not find evidence against normality, multicollinearity, or auto-correlation, or significant homoscedasticity. Data were analyzed using the software package $P$ sych ${ }^{23}$ for the statistical software, $R$ (version 3.2.5). ${ }^{24}$

\section{Conclusion}

Results of our study provided preliminary evidence for our hypothesis that posited an association between residents' report of having a supportive team preceptor and resident wellness, above and beyond self-care behaviors. Our study highlight the potential importance of supportive relationships within care teams on resident wellness. Our findings suggests that team leads may benefit from specific training on fostering supportive relationships with supervisees in the context of their leadership position and team-based care, as well as ongoing feedback from residents. Furthermore, residents may benefit from training during residency in order to promote a culture of supportive team leadership in the future.

Major limitations of our study include the cross-sectional nature of our data and the small sample size, both which significantly limit our power to detect smaller effect sizes and increase the risk for Type I and Type II errors and limit the generalizability of our findings. Given the exploratory nature of the current study, additional research is warranted to examine and replicate the nature of the relationship between preceptor team lead support and resident wellness in a larger sample. Furthermore, the causality of these relationships cannot be determined based on this cross-sectional design. Future research utilizing longitudinal and/or experimental designs would be helpful to clarify the direction of the relationship between variables.

In order to improve the quality and cost-effectiveness of healthcare and to support the Quadruple Aim, it is important to attend to understanding modifiable pathways that have the potential to increase the wellness of healthcare providers in team-based care. The current study suggests that among family medicine residents in an academic medical setting working in team-based care, positive relationships with their preceptor team-lead is associated with residents' wellbeing, beyond that of self-care behaviors. Although the focus of this study was on relationships with preceptor team leads, future research should also be conducted to explore additional relationships within care teams in academic medical settings (eg, with peers, support staff, interdisciplinary team members) and their influence on resident wellness.

\section{Tables and Figures}


Figure 1

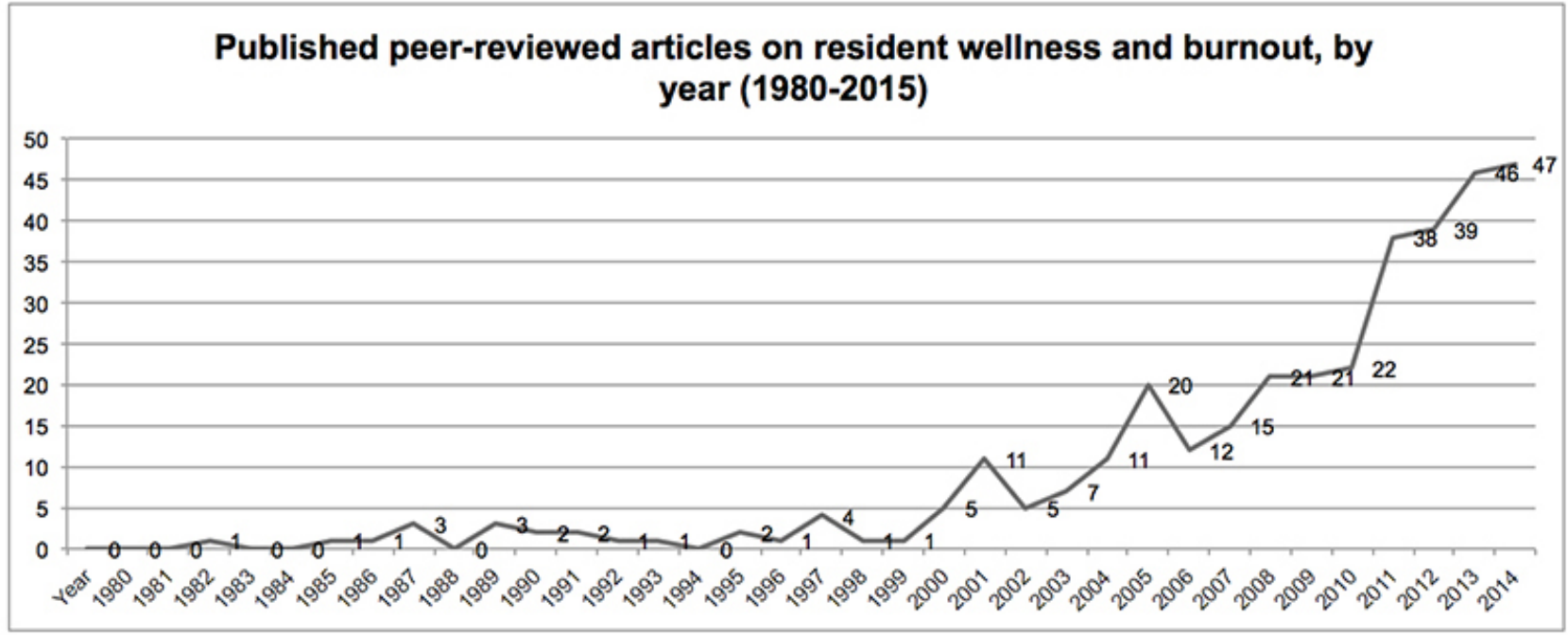

Table 1. Descriptive Statistics for Study Measures.

\begin{tabular}{|l|c|c|c|c|c|c|}
\hline \multicolumn{1}{|c|}{ Measures } & $\boldsymbol{M}$ (SD) & $95 \% \mathrm{Cl}$ of $\boldsymbol{M}$ & $\boldsymbol{M d n}$ & $\begin{array}{c}\text { Observed } \\
\text { Range }\end{array}$ & $\begin{array}{c}\text { Theoretical } \\
\text { Range }\end{array}$ & Cronbach's $\boldsymbol{\alpha}[\mathbf{9 5 \%} \mathrm{Cl}]$ \\
\hline Mindful Self-Care Scale & $100.5(18.90)$ & {$[93.95,107.05]$} & 99.5 & 70 to 154 & 33 to 165 & $.91[.86, .95]$ \\
\hline Team Lead Relationship & $5.93(.86)$ & {$[5.64,6.22]$} & 6 & 4 to 7 & 1 to 7 & $.92[.87, .96]$ \\
\hline Brief Resident Wellness & $21.26(3.40)$ & {$[20.13,22.39]$} & 22 & 13 to 30 & 5 to 35 & $.83[.72, .90]$ \\
\hline
\end{tabular}

Table 2. Kendall's Tau-b Bivariate Correlation Coefficients for Study Variables

\begin{tabular}{|l|c|c|c|c|c|}
\hline \multicolumn{1}{|c|}{ Study Variable } & Gender & $\begin{array}{c}\text { Postgraduate } \\
\text { Year }\end{array}$ & $\begin{array}{c}\text { Mindful Self-Care } \\
\text { Scale }\end{array}$ & $\begin{array}{c}\text { Team lead } \\
\text { Relationship }\end{array}$ & $\begin{array}{c}\text { Brief Resident } \\
\text { Wellness Profile }\end{array}$ \\
\hline Gender $^{\mathrm{a}}$ & - & -.05 & -.10 & .20 & $.43^{* *}$ \\
\hline Postgraduate year & & - & .18 & -.06 & .19 \\
\hline Mindful Self-Care Scale & & & - & .02 & .21 \\
\hline Team Lead Relationship & & & & - & $.40^{* *}$ \\
\hline Brief Resident Wellness Profile & & & & & - \\
\hline
\end{tabular}

${ }^{\circ}$ Gender Coded as: $0=$ Female; $1=$ Male. ${ }^{*} P<.05,{ }^{* *} P<.01$ 
Table 3. Summary of Hierarchical Linear Multiple Regression for Brief Resident Wellness Profile Score

\begin{tabular}{|c|c|c|c|c|c|c|c|}
\hline \multirow[b]{2}{*}{ Variable } & \multicolumn{4}{|c|}{ Coefficients } & \multicolumn{3}{|c|}{ Model Statistics } \\
\hline & $b$ & $\beta$ & $95 \% \mathrm{Cl}$ of b & $P$ Value & $R^{2}$ & Change in $R^{2}$ & $P$ Value \\
\hline Step 1 & & & & & .21 & - & .035 \\
\hline Postgraduate year & .99 & .24 & {$[-0.45,2.43]$} & .169 & & & \\
\hline Gender (male) & 2.89 & .42 & {$[0.53,5.26]$} & .018 & & & \\
\hline Step 2 & & & & & .34 & .13 & .027 \\
\hline Postgraduate year & .75 & .18 & {$[-0.62,2.10]$} & .273 & & & \\
\hline Gender (male) & 3.16 & .46 & {$[0.94,5.38]$} & .007 & & & \\
\hline Mindful Self-Care Scale & .07 & .37 & {$[0.01,0.13]$} & .027 & & & \\
\hline Step 3 & & & & & .50 & .16 & .007 \\
\hline Postgraduate year & 0.66 & 0.16 & {$[-5.50,11.35]$} & .268 & & & \\
\hline Gender (male) & 2.37 & .34 & {$[0.32,4.42]$} & .025 & & & \\
\hline Mindful Self-Care Scale & .06 & .32 & {$[0.01,0.11]$} & .029 & & & \\
\hline Team lead relationship & 1.69 & .42 & {$[.51,2.88]$} & .007 & & & \\
\hline
\end{tabular}

${ }^{a}$ Gender Coded as: $0=$ Female, $1=$ Male.

\section{Author Affiliations}

Cesar A. Gonzalez, PhD - Departments of Psychiatry, Psychology, and Family Medicine, College of Medicine, Mayo Clinic, Rochester, MN

Natalie E Gentile, MD - Department of Family Medicine, College of Medicine, Mayo Clinic, Rochester, MN Kurt B Angstman, MD - Department of Family Medicine, College of Medicine, Mayo Clinic, Rochester, MN

Julia R Craner, PhD - Division of Psychiatry and Behavioral Medicine, Spectrum Health Medical Group, and College of Human Medicine, Michigan State University, Grand Rapids, MI

Robert P. Bonacci, MD - Department of Family Medicine, College of Medicine, Mayo Clinic, Rochester, MN

\section{References}

1. Center C, Davis M, Detre T, et al. Confronting depression and suicide in physicians: a consensus statement. JAMA. 2003;289(23):3161-3166. doi:10.1001/jama.289.23.3161.

2. West CP. Physician Well-Being: Expanding the Triple Aim. J Gen Intern Med. 2016;31(5):458-459. doi:10.1007/s11606-016-3641-2.

3. Bodenheimer T, Sinsky C. From triple to quadruple aim: care of the patient requires care of the provider. Ann Fam Med. 2014;12(6):573-576. doi:10.1370/afm.1713.

4. Hull SK, DiLalla LF, Dorsey JK. Prevalence of health-related behaviors among physicians and medical trainees. Acad Psych. 2008;32(1):31-38. doi:10.1176/appi.ap.32.1.3.

5. Toker S, Biron M. Job burnout and depression:unraveling their temporal relationship and considering the role of physical activity. J Appl Psychol. 2012;97(3):699. doi:10.1037/e604062012-120.

6. Marchand A, Juster R-P, Durand P, Lupien SJ. Burnout symptom sub-types and cortisol profiles: What's burning most? Psychoneuroendocrinology. 2014;40:27-36. doi:10.1016/j.psyneuen.2013.10.001.

7. Kassam A, Horton J, Shoimer I, Patten S. Predictors of well-being in resident physicians: a descriptive and psychometric study. J Grad Med Educ. 2015;7(1):70-74. doi:10.4300/jgme-d-14-00022.1.

8. Min AA, Sbarra DA, Keim SM. Sleep disturbances predict prospective declines in resident physicians' psychological well-being. Med Educ Online. 2015;20. doi:10.3402/meo.v20.28530.

9. Shanafelt TD, Mungo M, Schmitgen J, Storz KA, Reeves D, Hayes SN, Sloan JA, Swensen SJ, Buskirk SJ. Longitudinal study evaluating the association between physician burnout and changes in professional work effort. In Mayo Clinic Proceedings 2016 Apr 30 (Vol. 91, No. 4, pp. 422-431). doi:10.1016/j.mayocp.2016.02.001.

10. Shanafelt TD, Dyrbye LN, West CP, Sinsky CA. Potential impact of burnout on the US physician workforce. In Mayo Clinic Proceedings 2016 Nov 1 (Vol. 91, No. 11, pp. 1667-1668). doi:10.1016/j.mayocp.2016.08.016. 
11. The Complexities of Physician Supply and Demand 2016 Update: Projections from 2014 to 2025. IHS Inc. www.aamc.org/download/458082/data/2016_complexities_of_supply_and_demand_projections.pdf. Accessed April 5, 2016.

12. Gupta R, Dubé K, Bodenheimer T. The road to excellence for primary care resident teaching clinics. Acad Med. 2016;91(4):458-461. doi:10.1097/acm.0000000000001100.

13. Grumbach K, Bodenheimer T. Can health care teams improve primary care practice? JAMA. 2004;291(10):1246-1251. doi:10.1001/jama.291.10.1246.

14. Bodenheimer T, Laing BY. The teamlet model of primary care. Ann Fam Med. 2007;5(5):457-461. doi:10/1370/afm.731.

15. Chen $\mathrm{EH}$, Thom DH, Hessler DM, et al. Using the teamlet model to improve chronic care in an academic primary care practice. J Gen Intern Med. 2010;25(4):610-614. doi:10.1007/s11606=010=1390-1.

16. Bodenheimer T, Willard-Grace R. Teamlets in primary care: enhancing the patient and clinician experience. J Am Board Fam Med. 2016;29(1):135-138. doi:10.3122/jabfm.2016.01.150176.

17. Martini S, Arfken CL, Churchill MA, Balon R. Burnout comparison among residents in different medical specialties. Acad Psychiatry. 2004;28(3):240-242. doi:10.1176/appi.ap.28.3.240.

18. Willard-Grace R, Hessler D, Rogers E, Dubé K, Bodenheimer T, Grumbach K. Team structure and culture are associated with lower burnout in primary care. J Am Board Fam Med. 2014;27(2):229-238. doi:10.3122/jabfm.2014.02.130215.

19. Sinsky CA, Willard-Grace R, Schutzbank AM, Sinsky TA, Margolius D, Bodenheimer T. In search of joy in practice: a report of 23 high-functioning primary care practices. Ann Fam Med 2013;11(3):272-278. doi:10.1370/afm.1531.

20. West CP, Dyrbye LN, Erwin PJ, Shanafelt TD. Interventions to prevent and reduce physician burnout: a systematic review and meta-analysis. The Lancet. 2016. doi:10.1016/s0140-6736(16)31279-x

21. Cook-Cottone C. The Mindful Self-Care Scale: Self-care as a tool to promote physical, emotional, and cognitive well-being. 2014. Retrieved from http://gse.buffalo.edu/about/directory/faculty/cook-cottone

22. Keim SM, Mays MZ, Williams JM, Serido J, Harris RB. Measuring wellness among resident physicians. Med Teach. 2006;28(4):370-374. doi:10.1080/01421590600625320.

23. Revelle W. An overview of the psych package. 2016. Retrieved from http://personality-project.org/r/overview.pdf.

24. R Core Team. R: A language and environment for statistical computing. R Foundation for Statistical Computing, Vienna, Austria. ISBN 3-900051-07-0, http://www.r-project.org; 2012.

Copyright $\odot 2017$ by the Society of Teachers of Family Medicine 